\title{
Underdetermination and Decomposition in Kepler's Astronomia Nova
}

\author{
By: Teru Miyake
}

Underdetermination and Decomposition in Kepler's Astronomia Nova Teru Miyake Nanyang Technological University One of the great difficulties of planetary astronomy at around the turn of the seventeenth century was that the observed two-dimensional motions of the planets across the celestial sphere are consistent with three different theories of the actual three- dimensional motions of the planets through space-the Ptolemaic theory, the Copernican theory, and the Tychonic theory. In other words, the theory of the actual motions of the planets was underdetermined by the available observations. In fact, as Kepler showed in the Astronomia Nova, by making minor modifications, you could make the theories empirically indistinguishable from each other, given the kinds of observations that were available at the time. It seemed to some astronomers in the sixteenth century that this underdetermination is unresolvable, and that, in fact, trying to determine the actual motions of the planets should not even be an aim of planetary astronomy.

The standard way to think about such cases of underdetermination is the following. We have a certain set of observations, in this case the two-dimensional motions of the planets across the celestial sphere. We have two or more sets of hypotheses, in this case the Ptolemaic theory, the Copernican theory, and the Tychonic theory. You deduce what observations you ought to see for each of the three theories, and then you compare these predicted observations with actual observations. Usually, you would accept the theory that has the best agreement between the predicted observations and the actual observations. But in some cases, more than one theory agrees with the observations just as well. These are cases of underdetermination. Philosophers have suggested ways of choosing between theories in cases of underdetermination, such as through so-called empirical virtues such as simplicity, but there is an undeniable arbitrariness in judging theories against such virtues.

I want to think about the problem of planetary astronomy, and certain cases of underdetermination, in a completely different way. We do not think of the problem as one of trying to find a theory that fits observations. Rather, we think of the solar system as what I call a complicated, partially inaccessible system. A complicated system is one that consists of many parts, those parts having various properties and relations with each other. I say a system is partially inaccessible if we can only confidently measure a proper part of the properties of, and relations between, the parts of that object. The solar system is obviously complicated in this sense-it consists of planets with various properties such as mass and relations such as planetary distances. In the seventeenth century, it was partially inaccessible. For example, the actual distances between the planets in three-dimensional space could not be known, because distances along the line of sight from the Earth could not be measured directly.

We can now think of the problem of planetary astronomy as one of determining a certain kind of inaccessible relation-actual distances between the planets in threedimensional space-from relations that are accessible to us, namely the twodimensional motions of the planets across the celestial sphere. Or, to put it another way, it is a problem in reconstructing three-dimensional motions from a twodimensional projection of those motions. When we project three-dimensional motion onto two dimensions, we are losing information, because a two-dimensional projection can be compatible with many different three-dimensional motions. That is, in fact, one way of thinking about the underdetermination between the Ptolemaic, Copernican, and Tychonic theories. 
In the Astronomia Nova, Kepler solves this problem by using the method of triangulation-setting up a triangle with the Sun, the Earth, and Mars at the corners and using geometrical relations to determine actual distances between the Earth and Mars. In order to determine these angles, however, it turns out that you must separate out the effects due to two different features of the planetary motions, known from the time of Ptolemy as the first inequality and the second inequality. The determination of the effects of the first and second inequalities is intertwined-in order to find out what the effect of the first inequality is, you must know the effect of the second inequality. In order to find out what the effect of the second inequality is, you must know the effect of the first inequality. Thus, there is a difficult problem of somehow separating out these intertwined effects.

More generally, I argue elsewhere that separating out effects that add together in this way, a process that I call decomposition, is actually an important part of acquiring knowledge about many partially inaccessible systems. In order to perform decomposition, you must make certain assumptions about the structure of the system. A question that often arises, then, is how to justify these assumptions.

I examine how Kepler carries out this process of decomposition in the Astronomia Nova, and then consider how the assumptions he uses are justified. I argue that the assumptions are ultimately justified by success in the very process of decomposition itself. For Kepler, the first inequality corresponds to features of the orbit of Mars, while the second inequality corresponds to the orbit of the Earth. A better determination of the Earth orbit allows a better determination of the Mars orbit, while a better determination of the Mars orbit allows a better determination of the Earth orbit. Success in further and further refining the orbits of Mars and the Earth is a good sign that the decomposition is on the right track.

This way of thinking about underdetermination provides a new understanding of how certain cases of underdetermination get resolved. By making certain assumptions about a system, measurements of theretofore inaccessible parts of the system could be enabled. These new measurements can, in some cases, resolve the underdetermination. The assumptions that enable the new measurements to be made are ultimately justified by success in carrying out the decomposition. Thus, the underdetermination is resolved by the enablement of new measurements through assumptions that are ultimately justified down the road. 